Hardy-Ramanujan Journal

Vol. 31 (2008)1-13

\title{
Ideal Solutions of the Tarry-Escott Problem of degree eleven with applications to
}

\section{Sums of Thirteenth Powers}

\section{Ajai Choudhry and Jarosław Wróblewski}

\begin{abstract}
This paper is concerned with the system of simultaneous diophantine equations $\sum_{i=1}^{6} A_{i}^{k}=\sum_{i=1}^{6} B_{i}^{k}$ for $k=2,4,6,8,10$. Till now only two numerical solutions of the system were known. This paper provides an infinite family of solutions. It is well-known that solutions of the above system lead to ideal solutions of the Tarry-Escott Problem of degree 11, that is, of the system of simultaneous equations, $\sum_{i=1}^{12} a_{i}^{k}=\sum_{i=1}^{12} b_{i}^{k}$ for $k=1,2,3, \ldots, 11$. We use one of the ideal solutions to prove new results on sums of $13^{\text {th }}$ powers. In particular, we prove that every integer can be expressed as a sum or difference of at most 27 thirteenth powers of positive integers.
\end{abstract}

Keywords: Tarry-Escott problem, multigrade equations, thirteenth powers, easier Waring problem.

2000 Mathematics Subject Classification: 11D41, 11D72, 11P05. 


\section{Introduction}

The first solution to the multigrade equation

$$
\sum_{i=1}^{6} A_{i}^{k}=\sum_{i=1}^{6} B_{i}^{k} \text { for } k=2,4,6,8,10
$$

was found in 1999 when Kuosa used a computer program written by Meyrignac to discover the equality

$$
151^{k}+140^{k}+127^{k}+86^{k}+61^{k}+22^{k}=148^{k}+146^{k}+121^{k}+94^{k}+47^{k}+35^{k}
$$

for $k=10$ and four days later Shuwen noticed that (2) holds for $k=2,4,6,8$ as well (see [4]). This was the only known solution until 2007 when Broadhurst [1] (see also [2]) discovered

$$
\begin{aligned}
& 2058^{k}+1896^{k}+1618^{k}+1109^{k}+891^{k}+257^{k} \\
= & 2037^{k}+1947^{k}+1514^{k}+1294^{k}+639^{k}+472^{k}, k=2,4,6,8,10 .
\end{aligned}
$$

In this paper we prove that the system (1) has infinitely many solutions and give a method to produce new numerical solutions.

This leads to infinitely many new ideal solutions of the Tarry-Escott Problem of degree 11, that is, of the system of simultaneous equations,

$$
\sum_{i=1}^{12} a_{i}^{k}=\sum_{i=1}^{12} b_{i}^{k} \text { for } k=1,2,3, \ldots, 11 .
$$

We use one of the solutions of (4) to obtain new results on sums of $13^{\text {th }}$ powers. In particular, we prove that every integer can be expressed as a sum or difference of at most 27 thirteenth powers of positive integers.

\section{A family of solutions}

In this Section we present a method of generating solutions to the diophantine system (1). 
We make the following substitutions

$$
\begin{aligned}
& A_{1}=2 x y+x z+2 y z-7 z^{2} \\
& A_{2}=2 x y-x z-2 y z-7 z^{2} \\
& A_{3}=2 x y-2 x z+y z+7 z^{2} \\
& A_{4}=2 x y+2 x z-y z+7 z^{2} \\
& A_{5}=\quad 3 x z+5 y z \\
& A_{6}=\quad 5 x z-3 y z \\
& B_{1}=2 x y+2 x z+y z-7 z^{2} \\
& B_{2}=2 x y-2 x z-y z-7 z^{2} \\
& B_{3}=2 x y-x z+2 y z+7 z^{2} \\
& B_{4}=2 x y+x z-2 y z+7 z^{2} \\
& B_{5}=\quad \begin{array}{c}
5 x z+3 y z \\
B_{6}=
\end{array} \quad 3 x z-5 y z
\end{aligned}
$$

and denote

$$
S_{k}=\sum_{i=1}^{6}\left(A_{i}^{k}-B_{i}^{k}\right) .
$$


It can be easily verified using Mathematica that

$$
\begin{aligned}
& S_{2}=S_{4}=0 \\
& S_{6}=4320 x y(x-y)(x+y) z^{4} f(x, y, z), \\
& S_{8}=5376 x y(x-y)(x+y) z^{4} f(x, y, z) \\
& \quad \times\left(8 x^{2} y^{2}+37 x^{2} z^{2}+37 y^{2} z^{2}+98 z^{4}\right), \\
& \quad \times\left(32 x^{4} y^{4}+248 x^{4} y^{2} z^{2}+665 x^{4} z^{4}+248 x^{2} y^{4} z^{2}+2384 x^{2} y^{2} z^{4}\right. \\
& \left.\quad+3038 x^{2} z^{6}+665 y^{4} z^{4}+3038 y^{2} z^{6}+4802 z^{8}\right) .
\end{aligned}
$$

where

$$
f(x, y, z)=8 x^{2} y^{2}-17 x^{2} z^{2}-17 y^{2} z^{2}+98 z^{4} .
$$

Hence any solution in integers of the equation

$$
8 x^{2} y^{2}-17 x^{2} z^{2}-17 y^{2} z^{2}+98 z^{4}=0
$$

leads by substitutions (5) to a solution in integers of the system (1).

\section{Solving $f(x, y, z)=0$}

In this Section we prove that equation (9) has infinitely many essentially distinct integer solutions and these solutions of (9) lead to infinitely many distinct and nontrivial solutions of (1).

On substituting

$$
x=U z, \quad y=V z /\left(8 U^{2}-17\right),
$$

equation (9) reduces to

$$
V^{2}=136 U^{4}-1073 U^{2}+1666,
$$


which is a quartic model of an elliptic curve. Four rational points on the curve (11) are easily seen to be $(U, V)=( \pm 1, \pm 27)$. On making the birational transformation defined by the relations

$$
\begin{aligned}
& U=(251 X+3 Y-206690) /(89 X+3 Y-36410) \\
& V=\left(243 X^{3}-766260 X^{2}+312978600 X-9256500 Y+59116365000\right) \\
& \quad \times(89 X+3 Y-36410)^{-2}
\end{aligned}
$$

and

$$
\begin{aligned}
& X=(-\left.86 U^{2}-1430 U+54 V+2974\right) /(U-1)^{2}, \\
& Y=\left(14688 U^{3}-57942 U^{2}-1602 U V-57942 U+4518 V+179928\right) \\
& \quad \times(U-1)^{-3},
\end{aligned}
$$

equation (11) reduces to the Weierstrass minimal form of the elliptic curve that is given by

$$
Y^{2}=X^{3}+X^{2}-1290080 X+556370100 .
$$

The rational point $(U, V)=(1,-27)$ on the curve $(11)$ corresponds to a rational point $P$ on the elliptic curve (14) which is given by

$$
(X, Y)=(9460 / 9,514250 / 27) \text {. }
$$

Since this rational point $P$ does not have integer co-ordinates, it follows from the Nagell-Lutz theorem on elliptic curves $[6$, p. 56] that this is not a point of finite order. Thus there exist infinitely many rational points on the elliptic curve (14), and these can be obtained by applying the group law. These infinitely many rational points on the curve (14) yield infinitely many rational solutions of equation (11). Finally using the relations (10), and assigning appropriate values to $z$ in each case, we get infinitely many integer solutions of equation (9). These integer solutions of equation (9) lead to infinitely many solutions of the diophantine system (1).

The point $P$ on the curve (14) leads to the solution $(x, y, z)=(1,3,1)$ of equation (9) which leads to a trivial solution of (1). To get nontrivial solutions 
of (1), we have to compute other points on the curve (14). For instance, the point $2 P$ given by $(X, Y)=(163579 / 225,-6090292 / 3375)$ on the curve (14) corresponds to the point $(U, V)=(-457 / 353,-1968867 / 124609)$ on the curve (11) which leads to the solution $(x, y, z)=(-101911,346293,78719)$ of (9) and finally to the following nontrivial solution of (1):

$$
\begin{aligned}
& \quad 1019171^{k}+774217^{k}+712866^{k}+447858^{k}+428496^{k}+102257^{k} \\
& =1018599^{k}+795069^{k}+652598^{k}+570049^{k}+264662^{k}+224448^{k}, \\
& k=2,4,6,8,10 .
\end{aligned}
$$

We shall now show that the above method actually generates infinitely many distinct and nontrivial solutions of (1). We observe that for any solution of (1) generated by a rational solution $(U, V)$ of equation (11), the ratio $A_{1} / A_{2}$ is determined only by $U$. For any given value of $\rho$, the condition $A_{1} / A_{2}=\rho$ may be considered as a polynomial equation in the single variable $U$ (after eliminating $V$ ), and this equation has only a finite number of rational roots. Similar remarks apply to all other ratios $A_{i} / A_{j}, A_{i} / B_{j}, B_{i} / B_{j}, \quad 1 \leq i \leq 6,1 \leq j \leq 6$. Any numerical solution of (1) fixes the ratios $A_{i} / A_{j}, A_{i} / B_{j}, B_{i} / B_{j}$, and hence can be generated only by a finite number of values of $U$. By the same logic, a finite set of distinct solutions of (1), together with the scalar multiples of these solutions, can be generated only by a finite number of rational values of $U$. Similarly the trivial solutions of (1), for which six of the ratios $A_{i} / B_{j}$ are \pm 1 , can be generated only by a finite number of rational values of $U$. As there are infinitely many rational solutions of equation (11), we are assured of generating infinitely many nontrivial and distinct solutions of (1) by the above method.

While the above method generates infinitely many solutions of (1), numerically small solutions can be found by searching for small solutions of (9) as is shown in the following Section. 


\section{Numerical solutions}

To obtain small solutions of the system (1) we search for small solutions of the equation (9). One way of setting up a relatively efficient search is to solve the equation (9) as a quadratic equation in $y$ and then search for $x, z$ yielding the discriminant being a square of an integer.

However, all the solutions we were able to find that way, can also be obtained by applying the group law to rational points on the elliptic curve (14), corresponding to two trivial solutions of (1).

Broadhurst's solution (3) can be obtained from $x=259, y=5607, z=178 \quad$ or $x=37, y=801, z=333$.

The smallest new solution we were able to find using the method presented in this paper is

$$
\begin{aligned}
& 1511^{k}+1138^{k}+1075^{k}+700^{k}+622^{k}+107^{k} \\
= & 1510^{k}+1180^{k}+953^{k}+886^{k}+413^{k}+293^{k}
\end{aligned}
$$

and it can be produced from $x=145, y=517, z=110$.

We were thus able to find 113 solutions with terms under $10^{100}$, including 8 solutions with terms under $10^{10}$ (5 solutions not mentioned so far are presented in Table I). 
Table I: Solutions of the Multigrade Equation (1)

\begin{tabular}{|r|r|r|r|r|r|}
\hline$A_{1}$ & 14770 & 23742 & 11603875 & 22263723 & 107581333 \\
$A_{2}$ & 12638 & 18687 & 8959475 & 19802832 & 80295173 \\
$A_{3}$ & 11632 & 18372 & 7853834 & 17523186 & 77895430 \\
$A_{4}$ & 7115 & 12734 & 5070974 & 11318686 & 52219540 \\
$A_{5}$ & 7043 & 9611 & 4404977 & 10177351 & 43290994 \\
$A_{6}$ & 929 & 349 & 1984396 & 2111827 & 4600567 \\
\hline$B_{1}$ & 14693 & 23708 & 11601341 & 22095904 & 107505355 \\
$B_{2}$ & 13165 & 19653 & 9033773 & 20464122 & 84106267 \\
$B_{3}$ & 10112 & 16426 & 7641076 & 15829981 & 66766060 \\
$B_{4}$ & 9718 & 14714 & 5725910 & 14318021 & 65824631 \\
$B_{5}$ & 4054 & 7713 & 3305329 & 6084678 & 30945298 \\
$B_{6}$ & 3455 & 3309 & 2713630 & 5604633 & 18205658 \\
\hline
\end{tabular}

\section{On Sums of thirteenth powers}

We can use solutions of (1) to prove certain results about sums of thirteenth powers. We will use the solution (2) of (1) as this is the smallest known numerical solution.

We use $\gamma=\gamma(k)$ to denote the least value of $n$ such that the equation

$$
x_{1}^{k}+x_{2}^{k}+\cdots+x_{m}^{k}=y_{1}^{k}+y_{2}^{k}+\cdots+y_{n}^{k},
$$

has infinitely many nontrivial primitive solutions in positive integers with $m<n$, that is, solutions such that $x_{i} \neq y_{j}, \quad 1 \leq i \leq m, 1 \leq j \leq n$, and $\operatorname{gcd}\left(x_{1}, x_{2}, \ldots, x_{m}, y_{1}, y_{2}, \ldots, y_{n}\right)=1$. 
We use $v(k)$ to denote the least value of $s$ such that every integer $n$ can be expressed in the form

$$
n=\varepsilon_{1} x_{1}^{k}+\varepsilon_{2} x_{2}^{k}+\ldots+\varepsilon_{s} x_{s}^{k}
$$

where $\varepsilon_{i}= \pm 1$ and $x_{i}$ is a positive integer or zero for all values of $i$.

Similarly, we use $g_{1}(k)$ to denote the least value of $s$ such that every rational number $r$ can be expressed in the form

$$
r=\varepsilon_{1} x_{1}^{k}+\varepsilon_{2} x_{2}^{k}+\ldots+\varepsilon_{s} x_{s}^{k},
$$

where $\varepsilon_{i}= \pm 1$ and all of the values of $x_{i}$ are rational.

We will now prove the following theorems about $13^{\text {th }}$ powers:

Theorem 1: $\gamma(13) \leq 13$.

Theorem 2: $v(13) \leq 27$.

Theorem 3: $g_{1}(13) \leq 24$.

All these theorems are new. It had been earlier proved that $v(13) \leq 39$ $[3$, p. 209] and this was subsequently improved to $v(13) \leq 31[5, \mathrm{p} .11]$. Analogues of Theorems 1 and 3 have not been published earlier.

We first prove the following identity:

$$
\begin{aligned}
& (x+151)^{13}+(x-151)^{13}+(x+140)^{13}+(x-140)^{13}+(x+127)^{13} \\
& +(x-127)^{13}+(x+86)^{13}+(x-86)^{13}+(x+61)^{13}+(x-61)^{13} \\
& +(x+22)^{13}+(x-22)^{13}-(x+148)^{13}-(x-148)^{13}-(x+146)^{13} \\
& -(x-146)^{13}-(x+121)^{13}-(x-121)^{13}-(x+94)^{13}-(x-94)^{13} \\
& -(x+47)^{13}-(x-47)^{13}-(x+35)^{13}-(x-35)^{13}=C_{1} x,
\end{aligned}
$$

where

$$
C_{1}=2^{14} \cdot 3^{10} \cdot 5^{3} \cdot 7^{2} \cdot 11^{2} \cdot 13^{3} \cdot 17 \cdot 19 \cdot 23 \cdot 29 \cdot 31 \text {. }
$$

It is easily seen that in the expansion of the left-hand side of (18), the coefficients of $x^{13}$ and even powers of $x$ are all 0 , and in view of the relations (2) where $k=2,4,6,8,10$, the coefficients of $x^{11}, x^{9}, \ldots, x^{3}$ also vanish. Thus 
the only nonzero term in the expansion is a term $C_{1} x$, and a little computation gives the coefficient of $x$ as stated in (19). This proves the identity (18).

In the identity (18) we substitute $x=C_{2} t^{13}$ where

$$
C_{2}=2^{12} \cdot 3^{3} \cdot 5^{10} \cdot 7^{11} \cdot 11^{11} \cdot 13^{10} \cdot 17^{12} \cdot 19^{12} \cdot 23^{12} \cdot 29^{12} \cdot 31^{12},
$$

when we get the following identity,

$$
\begin{aligned}
& \left(C_{2} t^{13}+151\right)^{13}+\left(C_{2} t^{13}-151\right)^{13}+\left(C_{2} t^{13}+140\right)^{13}+\left(C_{2} t^{13}-140\right)^{13} \\
& +\left(C_{2} t^{13}+127\right)^{13}+\left(C_{2} t^{13}-127\right)^{13}+\left(C_{2} t^{13}+86\right)^{13}+\left(C_{2} t^{13}-86\right)^{13} \\
& +\left(C_{2} t^{13}+61\right)^{13}+\left(C_{2} t^{13}-61\right)^{13}+\left(C_{2} t^{13}+22\right)^{13}+\left(C_{2} t^{13}-22\right)^{13} \\
& =\left(C_{2} t^{13}+148\right)^{13}+\left(C_{2} t^{13}-148\right)^{13}+\left(C_{2} t^{13}+146\right)^{13}+\left(C_{2} t^{13}-146\right)^{13} \\
& \left(C_{2} t^{13}+121\right)^{13}+\left(C_{2} t^{13}-121\right)^{13}+\left(C_{2} t^{13}+94\right)^{13}+\left(C_{2} t^{13}-94\right)^{13} \\
& +\left(C_{2} t^{13}+47\right)^{13}+\left(C_{2} t^{13}-47\right)^{13}+\left(C_{2} t^{13}+35\right)^{13}+\left(C_{2} t^{13}-35\right)^{13}+\left(C_{3} t\right)^{13},
\end{aligned}
$$

where

$$
C_{3}=2^{2} \text {.3.5.7.11.13.17.19.23.29.31. }
$$

It is readily seen that when $t$ is any positive integer, the above identity provides a solution in positive integers of the diophantine equation

$$
\sum_{i=1}^{12} x_{i}^{13}=\sum_{i=1}^{13} y_{i}^{13}
$$

This proves Theorem 1 .

Let $\Delta(k, m)$ be the smallest value of $s$ such that for every integer value of $n$ the congruence

$$
x_{1}^{k}+x_{2}^{k}+\ldots+x_{r}^{k}-x_{r+1}^{k}-\ldots-x_{s}^{k} \equiv n \quad(\bmod m),
$$

is solvable for some value of $r=r(n)$. Further, let $\Delta(k)=\max _{m} \Delta(k, m)$. Fuchs and Wright [3] have shown how the values of $\Delta(k, m)$ and $\Delta(k)$ may be calculated and, in fact, they have shown that $\Delta(13)=6$. 
Since there are $2413^{\text {th }}$ powers on the left-hand side of (18), it follows from this identity that

$$
v(13) \leq 24+\Delta\left(k, C_{1}\right)
$$

Following the method described in [3], the value of $\Delta\left(k, C_{1}\right)$ can be calculated, and in fact, we get

$$
\Delta\left(k, C_{1}\right)=3 .
$$

As the method given in [3] is based on a series of lemmas and theorems, we give below a direct computer assisted proof of (22) based on only the first lemma proved by Fuchs and Wright [3, Lemma 1, p. 192] which is as follows: Lemma 1: If $\operatorname{gcd}\left(m_{1}, m_{2}\right)=1$, and if, for given $n, s, r$, there is a solution of (20) when $m=m_{1}$ and a solution when $m=m_{2}$, then there is a solution when $m=m_{1} m_{2}$.

We will use this lemma with $r=s=3$ and $m=C_{1}$. We first show that the congruence

$$
x_{1}^{13}+x_{2}^{13}+x_{3}^{13} \equiv n \quad(\bmod m),
$$

is solvable for all possible values of $n$ and for all the coprime factors of $C_{1}$, namely

$$
2^{14}, 3^{10}, 5^{3}, 7^{2}, 11^{2}, 13^{3}, 17,19,23,29,31 .
$$

It is easily seen that if $\varphi(m)$ is coprime to 13 , then every integer coprime to $m$ is a $13^{\text {th }}$ power residue $\bmod m$. If moreover $m$ is a prime power, then every integer $n$ can be written as a sum of two $13^{\text {th }}$ power residues $\bmod m$, namely $n=n+0$ or $n=(n-1)+1$, depending on whether $n$ or $n-1$ is coprime to $m$. This argument does not apply when $m$ is a power of 13 .

To show that (23) is solvable when $m=13^{3}$, it suffices to take $0 \leq x_{i} \leq$ $168, i=1,2,3$ and write a computer program to work out all the residues of $x_{1}^{13}+x_{2}^{13}+x_{3}^{13}$ modulo $13^{3}$. It is then easily seen that there is a solution of (23) for all possible values of $n$ when $m=13^{3}$.

The congruence (23) is thus solvable for all values of $n$ when $m$ is any of the coprime factors listed in (24). It now follows from Lemma 1 that (23) is solvable for all values of $n$ when $m=C_{1}$, and hence $\Delta\left(k, C_{1}\right) \leq 3$. Further, it is readily verified by a computer program that the congruence

$$
x_{1}^{13}+x_{2}^{13} \equiv 5 \quad\left(\bmod 13^{3}\right),
$$


has no solution, thus $\Delta\left(k, C_{1}\right)>2$. This proves the relation (22).

It follows from the relations $(21)$ and $(22)$ that $v(13) \leq 27$. This proves Theorem 2.

Theorem 3 is an immediate consequence of the identity (18).

\section{$6 \quad$ Final remarks and acknowledgements}

Finding the set of substitutions (5) was possible by analysis of the numerical solution (3). We are grateful to David Broadhurst for making this paper possible by discovering this solution.

However the first known solution (2) cannot be obtained by substitutions (5) and we were unable to produce a set of similar substitutions leading to it.

\section{References}

[1] D. Broadhurst, A new Prouhet-Tarry-Escott solution, http://listserv.nodak.edu/cgi-bin/

wa. exe?A2=ind0709\&L=nmbrthry\&T=0\&P=R994

[2] D. Broadhurst, A Chinese Prouhet-Tarry-Escott solution, http://physics.open.ac.uk/ dbroadhu/cpte.pdf

[3] W. H. J. Fuchs and E. M. Wright, The 'easier' Waring problem, Quart. J. Math. (Oxford), 10 (1939), 190-209.

[4] J.-C. Meyrignac, Computing Minimal Equal Sums Of Like Powers, http://euler.free.fr/

[5] T. Rai, Easier Waring problem, The Journal of Scientific Research, Banaras Hindu University, J. Sci. Res. Banaras Hindu Univ. 1 (1950-51), $5-12$. 
[6] J. H. Silverman and J. Tate, Rational points on elliptic curves (Springer, New York, 1992).

Postal Address: Ajai Choudhry,

D-6/1, Multi-Storey Flats, Sector 13, R.K. Puram,

New Delhi-110066,

INDIA.

E-mail: ajaic203@yahoo.com

Postal Address 2: Jarosław Wróblewski,

Mathematical Institute, University of Wroclaw,

pl. Grunwaldzki 2/4,

50-384 Wrocław, POLAND.

E-mail: jwr@math.uni.wroc.pl

Received on 31-03-08

Accepted on 09-04-08 